\title{
Marketing forecasting based on Big Data information
}

\author{
Sergey Ivanov $^{1, *}$ and Mykola Ivanov ${ }^{1, * *}$ \\ ${ }^{1}$ Zaporizhzhia National University, 66 Zhukovsky Str., Zaporizhzhia, 69063, Ukraine
}

\begin{abstract}
In the paper discusses the use of big data as a tool to increase data transfer speed while providing access to multidimensional data in the process of forecasting product sales in the market. In this paper discusses modern big data tools that use the MapReduce model. The big data presented in this article is a single, centralized source of information across your entire domain. In the paper also proposes the structure of a marketing analytics system that includes many databases in which transactions are processed in real time. For marketing forecasting of multidimensional data in Matlab, a neural network is considered and built. For training and building a network, it is proposed to construct a matrix of input data for presentation in a neural network and a matrix of target data that determine the output statistical information. Input and output data in the neural network is presented in the form of a $5 \times 10$ matrix, which represents static information about 10 products for five days of the week. The application of the Levenberg-Marquardt algorithm for training a neural network is considered. The results of the neural network training process in Matlab are also presented. The obtained forecasting results are given, which allows us to conclude about the advantages of a neural network in multivariate forecasting in real time.
\end{abstract}

\section{Introduction}

As Laurent Flores explained [1], the success of digital marketing is determined by the success of digital marketing, thus how they are measured and used. However, attention is paid to marketing forecasting in the digital economy, taking into account intelligent systems. Intelligent systems and the use of multidimensional communication determined the emergence of a new concept by Klaus Schwab [2]. According to this concept, it is argued that we are in the era of the fourth industrial revolution (Industry 4.0), when the virtual world is combined with the physical world using information technology. The fourth industrial revolution is characterized by a change in economic relations and the widespread use of intelligent technologies (big data [3], artificial neural networks [4], and others).

It should be noted that with the use of digital marketing, D2C models have come to be used. The D2C (Direct to Consumer) model represents a direct selling system, where companies themselves manufacture, promote, sell and deliver their product without the involvement of intermediaries. So, the article provides an analysis that shows that, unlike traditional promotion through retail chains, companies using the D2C model develop faster with their own distribution channels [5]. Thanks to their good positioning, these companies not only have a competitive advantage in the market, but also have their own structure on the Internet. These companies have changed the producerconsumer relationship and are reducing the distance be-

\footnotetext{
*e-mail: flydaiver@gmail.com

**e-mail: nn_iva@ukr.net
}

tween them. Today, any customer can contact the manufacturer directly, ask their question and make a purchase, avoiding extra charges and saving time. Renowned manufacturers have recognized the need to develop their own D2C strategies based on marketing analytics. The authors of the article acknowledge that the use of D2C opens up additional opportunities for companies. According to the authors, Nike is a prime example, with D2C sales accounting for a third of total revenues by the end of 2020 based on its Consumer Direct strategy.

Given the widespread use of digital marketing, Rimma Katz in article [6] explored social commerce, which fostered development along the D2C model. Today, social commerce is used to increase the reach of consumers, those who may know about direct contact with the manufacturer.

So in the presented report by Jasmine Enberg [7], a summary of the main events, their analysis for marketing management, solving problems strategic development of companies. Report author Jasmine Enberg determines that the global forecast for monthly social media users in 2020 has increased due to the effects of the pandemic. However, no platform will be able to maintain the growth it picked up at the beginning of the year. Therefore, in 2021, the growth rate will begin to normalize. Recent product launches, including Facebook live shopping and Instagram shopping tags, show that e-commerce continues to be a priority for the two platforms. Snapchat and Twitter were more focused on effective marketing, namely the release of new sets of promotional offers with direct consumer response and others. 
Therefore, the modern market is based on direct selling (D2C) models. allow you to get images of buyers and segment them. In addition to these tasks, it is necessary to solve forecasting the market, which changes every year.

It should be noted that worldwide targeted statistics for the entire sales system. This information is stored in cloud storage (Big Data). The information used includes data from the time of attracting a new consumer to the required resource information about the number, including repeated ones.

In this article, we propose a marketing analytics method based on artificial neural networks.

Marketing forecasting is part of any firm's overall marketing analytics. An important role in the forecasting method is played by the multidimensionality of information and methods of their processing. The use of Big Data with OLAP technologies requires new approaches to processing and applying large amounts of data. This is due to the wide range of communication systems used in the e-commerce market. Therefore, marketing forecasting based on Big Data is an urgent task, which is discussed in this article.

The article is devoted to marketing forecasting based on the use of neural networks. The information base of marketing forecasting is Big Data. The process of constructing training matrices, training a neural network and making the predictions are presented in the result of paper.

\section{Literature review}

Modern companies use many available forecasting methods, they not only improve the quality of their products, but also get information about the needs of customers. Marketing forecasting models are a great way to predict customer preferences and apply new ways to stand out from the competition. Using practical forecasting models today is the best way to get the most effective and complete data to improve marketing decisions. In this case, forecasting methods in digital marketing should include not only customer surveys, their age, interests and price, but also the characteristics of the product, brand, logistics and others.

In the work of Yonathan Bard [8], the problem of fitting mathematical models to numerical data was considered. Such a fit is often performed by the least squares method, regardless of previous knowledge of parameter values or the statistical nature of measurement errors. According to the results of a study of the opinions of experts, Alison Hubbard Ashton [9] considered a scenario in which the results of the opinions of experts differ significantly from the polls of intentions. For this case, the author considered the problem of predicting market behavior.

Further development of the principle of intentions was found in the work of Vicki G. Morwitz [10]. The author proposed the principles of using intentions when solving the forecasting problem. These tasks included researching people and how they would behave in different situations. For this, the method of polling the intentions of people was used. Intent surveys are widely used in marketing when sales data is unknown, for example, to forecast new products.

A continuation of these works found themselves in the work of J. Scott Armstrong [11], where the role of a person as a dominant factor was considered. This task was solved as a role-playing game for making predictions of the behavior of people who interact with others. A key tenet of this approach is to provide realistic simulation of interactions.

This forecasting method is currently rarely used. Gene Rowe and George Wright [12] considered the application of the Delphi method as a procedure. The authors found that the accuracy of expert predictions can be improved through the use of Delphi structured methods. One of the principles of the method is that experts' forecasts should not depend on each other. Expert groups sometimes violate this principle; as a result, the data should not be used in forecasting.

The task "Intentions" was considered in their work by Dick R. Wittink and Trond Bergestuen [13]. This paper examines the intention as an indicator of a consumer to purchase a product under the influence of various factors. The consumer can declare his intentions to make a purchase of various goods. This method is based on the following principles, namely, using a new design to create an acceptable situation.

The formation of a digital marketing strategy was considered in the work of Pinaki Mandal and Nitin Joshi [14]. The authors of the article emphasize that digital technologies make marketing more effective, since they allow to identify individual consumer interests, better manage campaigns and improve the product. In this article, the authors propose a flowchart for developing marketing strategies.

The analysis of the state of the digital economy and digital marketing is discussed in the article by Mykola Ivanov [15]. The author in the article shows that the dynamics of processes in the economy is quite high and requires a quick analysis of multidimensional data. The author proposes a conceptual model and a method for assessing consumer demand in the target market, aimed at the prospective management of trading floors using Big Data.

The analysis of the overview of macroeconomic forecasting are discussed in the [16-19]. The authors take the focus on a wide range of theories as well as empirical methods: business cycle analysis, time series methods, macroeconomic models, medium and long-run projections, fiscal and financial forecasts, and sectoral forecasting.

The research of the technological sources of the next long wave of growth is made in $[20,21]$. The authors study the impacts of national innovation systems (NIS) and economic complexity index (ECI) on economic growth.

The use of Exponential smoothing (ES) forecasting methods is discussed in $[22,23]$. The authors research revolution of exponential smoothing, which has been improved with the introduction of a complete modeling framework incorporating innovations state space models, likelihood calculation, prediction intervals and procedures for model selection. 


\section{Marketing forecasting modeling}

The modern economy is characterized by rapid dynamics of economic processes. Under these conditions, marketing forecasting models acquire new meanings in managerial decision-making. The process of the importance of making management decisions in digital marketing systems is shown in figure 1.

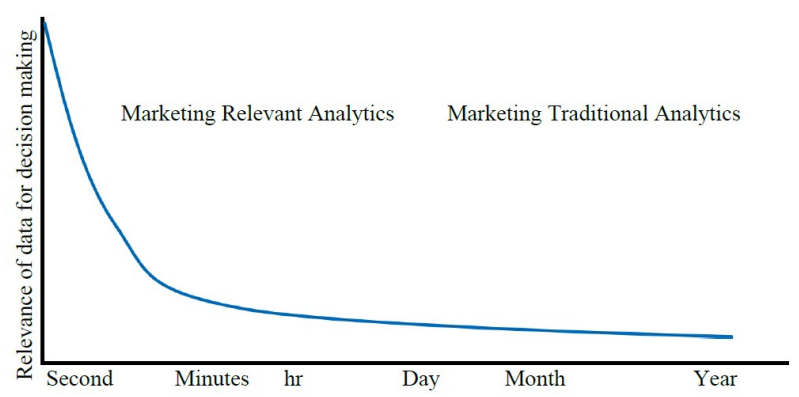

Figure 1. The importance of management decision making in digital marketing

Traditional Marketing Analytics (MTA) is based on the use of classical approaches and forecasting methods. MRA is aimed at solving economic important forecasting problems from the moment information appears to several hours.

These tasks are solved using neural networks and marketing robots.

Traditional Marketing Analytics is based on the use of classical approaches and forecasting methods. Marketing Actual Analytics (MRA) is aimed at solving economic important forecasting problems from the moment information appears to several hours. These tasks are solved using neural networks and marketing robots. Today the number of information sources of data in the world is growing rapidly. Therefore, storage technologies and their processing of information are becoming more and more in demand. By storing information, one can single out the use of Big Data for which the basic principles of work can be formulated:

1. Horizontal scalability, which takes into account that the data can be arbitrarily large from any system. They have the ability to handle big data.

2. Tolerance to failures, which use the principles of horizontal scalability and apply methods of clustering systems; locality of data, which allows in large distributed systems to separate data from a large number of data centers. All modern tools for working with big data, one way or another, follow these three principles. The first principle is based on the MapReduce model. The MapReduce model provides for distributed data processing proposed by Google and is shown in figure 2.

MapReduce provides that data is organized as relational or multidimensional data (OLAP). The data processing method takes place in three stages. The first stage is

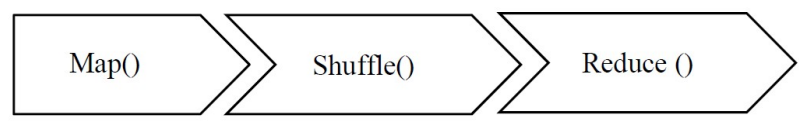

Figure 2. Data processing according to the MapReduce model

aimed at executing the Map() function. At this stage, the data is preprocessed using the $\operatorname{Map}()$ function, which is defined by the user. The work of this stage is to preprocess and filter the data. The second stage of the model is performed by the Shuffle() function. This stage goes unnoticed by the user. At this stage, the Map() function performs the data immersion procedure similarly to the formation of data marts (Data Mart), that is, one Map() data output corresponds to each mart. In the future, these showcases will serve as an input for the Reduce() function. The third stage of the model is aimed at executing the Reduce() function. Each data mart, which is formed in the second stage, transfers information to the input of the Reduce() function. The Reduce() function is user defined and calculates the result for individual storefronts. The set of all values returned by Reduce() is the result in this method. Therefore, Big Data technology is consider as a tool that allows you to increase the speed of data transfer while providing a large capacity of information carriers. In addition, this technology can improve the availability of cloud applications and data services. Thus, digital marketing is shape around the mainstream e-commerce models. The interconnection of the main models (B2B, B2A, D2C, C2A and C2C) of e-commerce systems based on the systems for collecting, storing and analyzing information in real time. Which based on subsequent storage in historical data layers. For the implementation of systems that perform Marketing Relevant Analytical tasks using data, OLAP data systems are used, which are structured according to the principle of multidimensional information presentation [15]. Reducing the cost of creating multidimensional warehouses can be achieve by using Data Mart. A data mart can only contain thematically aggregated data. Big Data is today a single, centralized source of information for the entire subject area. The structure of the marketing analytical system can be represent as follows (figure 3).

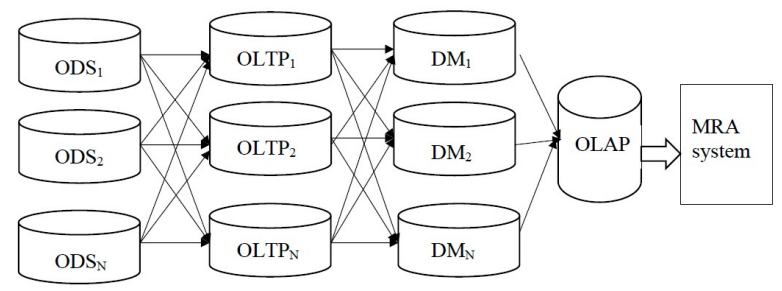

Figure 3. The structure of the marketing analytical system

In a marketing analytical system, there are many databases. where transaction processing is done in real time. Therefore, online data source systems (ODS) pro- 
vide information for processing in OLTP. OLTP systems provide storage and processing of information in real time. The processed data in OLTP is transferred to the Data Mart systems with the subsequent construction of multidimensional OLAP data cubes.

This multidimensional data is aimed at presenting information on thematic sections both on marketing information and other information from different areas of the economy. The marketer has the ability to access multidimensional data in the repository, as well as complete economic information for conducting an MRA. The advantages of this approach are:

- simplicity of creating and filling OLAP, since filling comes from reliable sources of data marts;

- reducing the load on working with multidimensional data, namely, one multidimensional query processes multiple OLAP layers.

The data coming from the OID is transferred to the OLTP and the data marts are moved. OLAP stores data as multidimensional layers of measures and dimensions [15]. For marketing forecasting, a multidimensional query is formed to multidimensional data, which allows obtaining the following information input stream (Inflow) - formed by data from OLTP and DMN subsystems:

$$
I=\left\{p_{j}=\left(g_{j}, i n_{j}, m i_{j}\right)\right\}, j=1, . ., N,
$$

where $g_{j}$ is the product included in the analyzed sets of $N$ - the object of research,

$i n_{j}$ - indicator of income $j$ of the product,

$m i_{j}$ - product marketing indicators $j$.

Datasets from set $I$ stored in OLAP and on demand allow you to obtain and conduct marketing analysis with the subsequent storage of data, which are called transactions. Description of a transaction to set $I$ as follows:

$$
T=\left\{i n_{j} \mid i n_{N} \in I\right\}
$$

Such transactions for retail outlets on the Internet correspond to the nomenclature of goods that the consumer buys and the data stored in OLAP as multidimensional data cubes (OLAP).

Then solving the problem of marketing forecasting based on a neural network, data arrays of its training are formed. The forecasting technique using a neural network is formalized through the problem of pattern recognition. Data on the predicted economic indicators of a product for a certain period of time form an image, the class of which is determined by the values of the predicted indicators.

In the proposed methodology, the dimension of the multidimensional array will determine both the forecasting interval and the number of predicted indicators. Each next line of the array is formed as a result of a shift by one interval equal to the prediction interval.

The neural network is trained on the generated training array of product indicators and adjusts its weights accordingly. As a result, the neural network is trained to solve the forecasting problem for a certain forecasting horizon. It should be noted that two forecasting approaches are used: one-step and multi-step. One-step forecasting is used for short-term forecasts and multistep forecasting is used for long-term forecasting.

Let the time interval $\left[t_{0}, t_{k}\right]$ be given, the indicators $g_{j}$, $i n_{j}, p r_{j}$ of the product are defined, where $t_{0}$ is the initial time value, $t_{k}$ is the current time value. To find the predicted values on the prediction interval $\Delta$, a method is proposed that includes the following stages:

1. Analytical analysis of marketing indicators and the formation of a learning matrix from selected values from historical slices of multidimensional databases (OLAP technology).

A learning matrix (ML - matrix learning) can be written as input data for representation in a neural network (equation (3)).

The input data in the neural network in Matlab is presented in the form of a $5 \times 10$ matrix, which represents static information on 10 products for five days of the week and has the following form (figure 4).

Target data that determine the output statistical information for the neural output can be represented in the form of learning value matrix (TVM $M_{\text {Output }}$, equation (4)).

The target data, which determines the output statistical information in Matlab for neural output, is presented in figure 5.

2. Neural network training. The process of training a neural network is to match to each $M L_{I}$ nput element the value of the $T V M_{O}$ utput matrix corresponding to the mapping in the value of the elements of the weight matrix $w_{j}$ :

$$
w_{j}: M L_{\text {Input }} \rightarrow T V M_{\text {Output }} .
$$

In the process of training the neural network, the task of minimizing the objective function is solved. With this approach, an algorithm is used for training, which is the most efficient not only in terms of errors, but also in time. The neural network in Matlab is trained using the Levenberg-Marquardt error backpropagation algorithm. The Levenberg-Marquardt algorithm uses a scalable conjugate gradient backpropagation. Therefore, the training of the neural network is represented in time, and the network is tuned in accordance with its error. The magnitude parameter is used to measure the generalization of the neural network and stop learning when the generalization stops improving. The test score itself does not affect training and provides an independent assessment of the performance of the neural network during and after training. The choice of the algorithm, as well as the learning process of the neural network, is shown in figure 6 and 7 .

The number of neural network training epochs can be written as follows:

$$
\text { epochs }=\left|\frac{t_{k}-t_{0}}{\Delta}\right| .
$$

In the process of forecasting by a neural network, it is necessary to take into account the forecasting horizon. In the Matlab system, the sim (net,[;;]) function is implemented, which allows you to supply a variety of input values and get a solution at the output of a neural network. The forecast results for the sale of 10 goods are shown in figure 8 . 


$$
M L_{\text {Input }}=\left[\begin{array}{cccc}
i n_{01}=f_{01}\left(t_{0}\right) & i n_{02}=f_{02}\left(t_{0}+\Delta\right) & \ldots & i n_{0 m}=f_{0} m\left(t_{0}+(k-1) \Delta\right) \\
i n_{11}=f_{11}\left(t_{0}\right) & i n_{12}=f_{12}\left(t_{0}+\Delta\right) & \ldots & i n_{1 m}=f_{1} m\left(t_{0}+(k-1) \Delta\right) \\
\ldots & \ldots & \ldots & \ldots \\
i n_{N 1}=f_{N 1}\left(t_{0}\right) & i n_{N 2}=f_{N 2}\left(t_{0}+\Delta\right) & \ldots & i n_{N m}=f_{N} m\left(t_{0}+(k-1) \Delta\right)
\end{array}\right], m=1, \ldots, k
$$

where $\Delta-$ is the horizon (time interval) of forecasting.

$$
T V M_{\text {Output }}=\left[\begin{array}{cccc}
i n_{01}=f_{01}\left(t_{0}+\Delta\right) & i n_{02}=f_{02}\left(t_{0}+2 \Delta\right) & \ldots & i n_{0 m}=f_{0 m}\left(t_{0}+k \Delta\right) \\
i n_{11}=f_{11}\left(t_{0}+\Delta\right) & i n_{12}=f_{12}\left(t_{0}+2 \Delta\right) & \ldots & i n_{1 m}=f_{1 m}\left(t_{0}+k \Delta\right) \\
\ldots & \ldots & \ldots & \ldots \\
i n_{N 1}=f_{N 1}\left(t_{0}+\Delta\right) & i n_{N 2}=f_{N 2}\left(t_{0}+2 \Delta\right) & \ldots & i n_{N m}=f_{N m}\left(t_{0}+k \Delta\right)
\end{array}\right], m=1, \ldots, k
$$

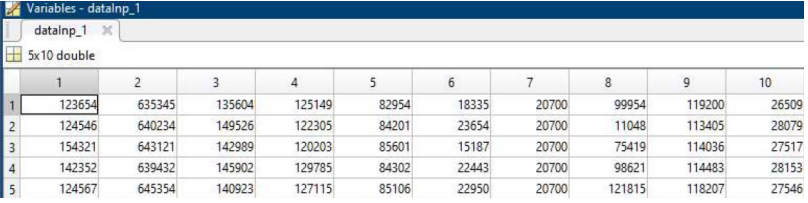

Figure 4. DataInp matrix view

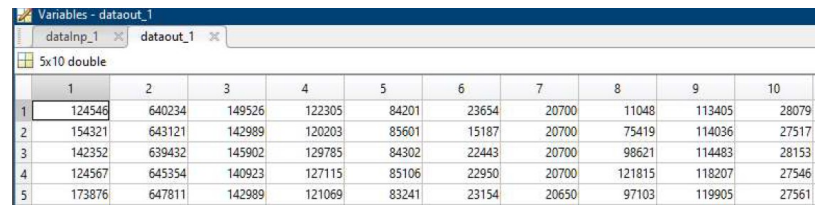

Figure 5. DataOut matrix view

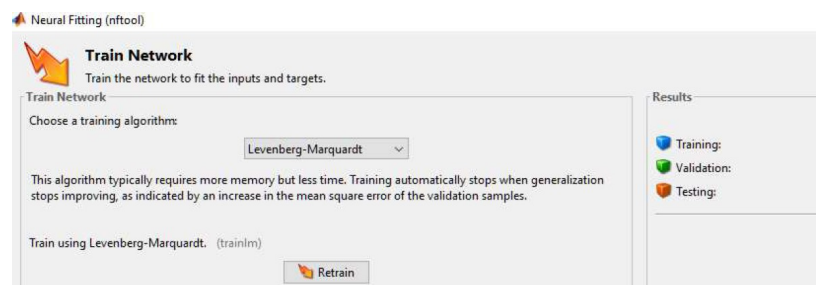

Figure 6. The choice of the learning algorithm

Therefore, the created neural network does indeed make multiple predictive decisions. It allows you to solve the sales function of marketing and consider the dynamics of the sale of many products in real time. Marketing forecasting looks at the number of future periods that the forecast will cover. That is, you may need a forecast 7 days ahead, with data for every day. In this case, the period is a day, and the horizon is 7 days. Finally, the prediction interval is the frequency with which a new prediction is made. Often the prediction interval coincides with the prediction period. The choice of the forecasting period and horizon is usually dictated by the conditions for making marketing decisions. Choosing these two parameters is one of the hardest parts of marketing forecasting. For forecasting to be meaningful, the forecasting horizon must be no less than the time required to implement the decision made on the basis of the forecast.

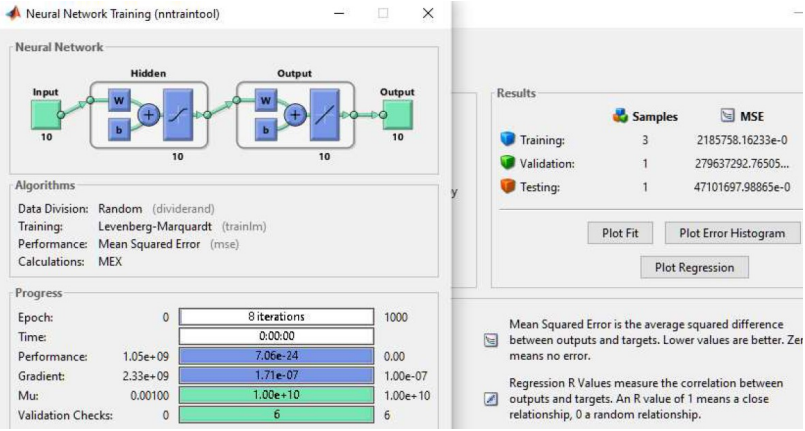

Figure 7. The process of learning the neural network

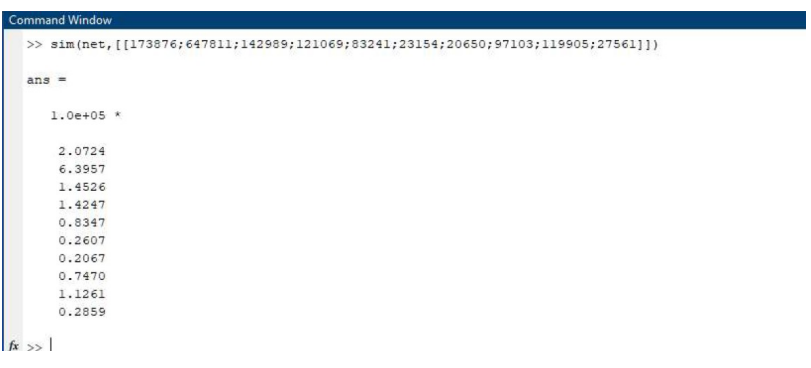

Figure 8. The result of forecasting the sale of 10 products

Thus, forecasting is highly dependent on the nature of the decision being made.

\section{Conclusion}

It is known that making management decisions in digital marketing, taking into account the time of receipt and a large amount of information are an urgent task today.

In addition, in the article, the authors consider the use of Big Data as a tool to increase the data transfer speed while providing access to multidimensional data (OLAP).

The article proposes the structure of the marketing analytics system. It includes many databases, where transactions are processed in real time. Consequently, online data source systems (ODS) provide information for processing in OLTP. This ensures prompt processing of information in real time. For marketing forecasting of multidimensional data, a neural network in Matlab is built. To solve the problem of improving forecasts, the authors have 
proposed building input data matrices for presentation in a neural network and target data matrices that determine the output statistical information.

Also in the paper, the results of the neural network training process in Matlab are represented on a chosen learning algorithm. The obtained forecasting results allow us to draw a conclusion about the advantages of a neural network in multivariate forecasting in real time. Multidimensional data and the level of their detail are important to solving the problem of forecasting in real time.

The wider use of digital-marketing systems gives an opportunity for applying the proposed approach to marketing forecasting.

\section{References}

[1] L. Flors, How to Measure Digital Marketing (Springer, London, 2014)

[2] K. Schwab, The Fourth Industrial Revolution (World Economic Forum, Cologny/Geneva, 2017)

[3] M. Ivanov, S. Ivanov, O. Cherep, N. Terentieva, V. Maltiz, I. Kaliuzhna, V. Lyalyuk, CEUR Workshop Proceedings 2713, 295 (2020)

[4] S. Semerikov, H. Kucherova, V. Los, D. Ocheretin, CEUR Workshop Proceedings 2845, 22 (2021)

[5] Insider Intelligence Editors, Nike's D2C sales will comprise a third of its total revenues (2021), https : //tinyurl. com/3dwxndf9

[6] R. Kats, Mucinex eyes social commerce to help bolster D2C business (2020), https: //tinyurl . com/ zzsm5zea

[7] J. Enberg, US Digital Ad Spending 2019 (2019), https://www . emarketer. com/content/ us-digital-ad-spending-2019

[8] Y. Bard, Nonlinear Parameter Estimation, 4th edn. (Academic Press, New York, NY, 1973)

[9] A.H. Ashton, The Accounting Review 60, 173 (1985)

[10] V.G. Morwitz, Methods for Forecasting from Intentions Data (Springer US, Boston, MA, 2001), pp.
33-56, ISBN 978-0-306-47630-3, https://doi. org $/ 10.1007 / 978-0-306-47630-3 \_3$

[11] J.S. Armstrong, Role Playing: A Method to Forecast Decisions (Springer US, Boston, MA, 2001), pp. 1530, ISBN 978-0-306-47630-3, https: //doi .org/ 10. $1007 / 978-0-306-47630-3 \_2$

[12] G. Rowe, G. Wright, in Principles of Forecasting: A Handbook for Researchers and Practitioners, edited by J.S. Armstrong (Springer US, Boston, MA, 2001), pp. 125-144, ISBN 978-0-306-47630-3, https:// doi .org/10.1007/978-0-306-47630-3_7

[13] D.R. Wittink, T. Bergestuen, Forecasting with Conjoint Analysis (Springer US, Boston, MA, 2001), pp. 147-167, ISBN 978-0-306-47630-3, https : //doi . org $/ 10.1007 / 978-0-306-47630-3 \_8$

[14] P. Mandal, N. Joshi, International Journal of Scientific Research and Management 5, 5428 (2017)

[15] M. Ivanov, CEUR Workshop Proceedings 2422, 395 (2019)

[16] N. Carnot, V. Koen, B. Tissot, Economic Forecasting (Palgrave Macmillan UK, London, 2005)

[17] N. Carnot, V. Koen, B. Tissot, Economic Forecasting and Policy (Palgrave Macmillan UK, London, 2011)

[18] S. Ivanov, CEUR Workshop Proceedings 2422, 385 (2019)

[19] C.P. Lim, ed., Handbook on Decision Making, Vol. 1: Techniques and Applications (Springer-Verlag, Berlin Heidelberg, 2010)

[20] D. Vázquez, Journal of Evolutionary Economics 30, 949 (2020)

[21] K. Lee, J. Lee, Journal of Evolutionary Economics 30, 897 (2020)

[22] C. Chatfield, A.B. Koehler, J.K. Ord, R.D. Snyder, Journal of the Royal Statistical Society: Series D (The Statistician) 50, 147 (2001)

[23] R. Hyndman, A. Koehler, K. Ord, R. Snyder, Forecasting with Exponential Smoothing: the State Space Approach (Springer, New York, 2008) 